\title{
Influencia de los hábitos orales en el perfil miofuncional orofacial de niños de tres a cinco años.
}

\author{
Raquel Andressa dos Santos Barraza, ${ }^{1}$ \\ Mônica Carminatti, ${ }^{2}$ \\ Aline Carneiro, ${ }^{1}$ \\ Bárbara de Lavra Pinto Aleixo, ${ }^{3}$ \\ Erissandra Gomes. ${ }^{4}$
}

\section{Resumen}

Objetivo: Investigar la influencia de los hábitos orales en el perfil miofuncional de los niños de tres a cinco años. Material y métodos: Estudio transversal con 73 niños a través del protocolo de aplicación validado para la evaluación de los aspectos miofuncional orofacial y el cuestionario sobre los hábitos orales. Para el análisis de las variables, el nivel de significación fue del 5\%. Resultados: La suma total de protocolo de Evaluación Miofuncional Orofacial con Valores (AMIOFE) fue de 91,6 \pm 5,4 puntos (IC del 95\%: 90,3 - 92,9), con un mínimo de 75 y un máximo de 100 puntos. Se observó relación significativa entre: la alimentación con biberón $(p=0,014)$ y chupetes $(p<0,001)$ con los aspectos y las posiciones de los articuladores; el uso del chupete y la función respiratoria $(p=0,04)$ y la deglución $(p=0,006)$; el uso de objetos en la boca y la función de masticación $(p=0,001)$; la respiración oronasal y los aspectos y las posiciones de los articuladores $(p<0,001)$ y la función de deglución ( $p=0,002)$. Además, la lactancia materna prolongada influyó positivamente el aspecto y la posición de los articuladores $(p=0,001)$ y la función respiratoria $(p=0,005)$. Conclusiones: Los hábitos de alimentación con biberón, los objetos en la boca, los chupetes y la respiración oronasal se relacionaron con cambios en los aspectos y posturas de los articuladores y las funciones estomatognáticas.

Palabras clave: Fonoaudiología, Hábitos, Niño, Preescolar, Sistema Estomatognático

Fonoaudióloga. Universidad Federal do Rio Grande do Sul (UFRGS), Graduada en Fonoaudiología. Porto Alegre, Rio Grande do Sul, Brasil.

Alumna de Doctorado en Clínica Odontológica. Universidad Federal do Rio Grande do Sul (UFRGS), Programa de Posgrado en Odontología. Porto Alegre, Rio Grande do Sul, Brasil. Becaria de la Coordenação de Aperfeiçoamento de Pessoal de Nível Superior (CAPES).

Doctora en Letras. Universidad Federal do Rio Grande do Sul (UFRGS), Graduada en Fonoaudiología. Porto Alegre, Rio Grande do Sul, Brasil.

4 Doctora en Ciencias Médicas. Universidad Federal do Rio Grande do Sul (UFRGS), Profesora de Grado de Fonoaudiología. Porto Alegre, Rio Grande do Sul, Brasil. 
Artigo original

\section{Influência dos hábitos orais no perfil miofuncional orofacial de crianças de três a cinco anos.}

\section{Resumo}

Objetivo: Verificar a influência dos hábitos orais no perfil miofuncional orofacial de crianças de três a cinco anos. Material e métodos: Estudo transversal, realizado com 73 crianças por meio da aplicação de protocolo validado para avaliação do aspecto miofuncional orofacial e o questionário sobre os hábitos orais. Para a análise entre as variáveis, o nível de significância utilizado foi de $5 \%$. Resultados: A soma total do protocolo Avaliação Miofuncional Orofacial com Escores (AMIOFE) foi de 91,6+5,4 pontos (IC95\%=90,3 - 92,9), com o mínimo de 75 e máximo de 100 pontos. Foi observada relação significativa entre: o uso da mamadeira $(p=0,014)$ e chupeta $(p<0,001)$ com os aspectos e posturas dos órgãos fonoarticulatórios; uso da chupeta e as funções de respiração $(p=0,04)$ e deglutição ( $p=0,006)$; o uso de objetos na boca e a função de mastigação $(p=0,001)$; respiração oronasal e aspectos e posturas dos órgãos fonoarticulatórios $(\mathrm{p}<0,001) \mathrm{e}$ função de deglutição $(p=0,002)$. Também, ter recebido aleitamento materno por mais tempo influenciou positivamente o aspecto e postura dos órgãos fonoarticulatórios $(p=0,001)$ e a função de respiração $(p=0,005)$. Conclusão: Os hábitos de mamadeira, objetos, chupeta e respiração oral estiveram relacionados a alterações nos aspectos e posturas dos órgãos fonoarticulatórios e nas funções estomatognáticas.

Palavras-chave: Fonoaudiologia, Hábitos, Criança, Pré-Escolar, Sistema Estomatognático.

Original article

\section{Influence of oral habits on the myofunctional orofacial profile of children aged three to five years-old.}

\section{Abstract}

Objective: Verify the influence of oral habits on miofunctional orofacial profile of children aged three to five yearsold. Material and methods: This cross sectional study included 73 children which underwent the application of validated protocol for evaluation of their myofunctional orofacial functions and a questionnaire about their oral habits.
A significance level of 5\% was set for the statistical analysis. Results: The Orofacial Myofunctional Evaluation with Scores (OMES) total sum was 91.6+5.4 points $(\mathrm{CI} 95 \%=90.3-92.9)$, with a minimum of 75 and maximum of 100 points. A significant association was identified between bottlefeeding $(p=0.014)$ and pacifier $(p<0.001)$ with respect to phonoarticulatory organs' appearance and posture, pacifier use and breathing $(p=0.04)$ and swallowing 
functions ( $p=0.006)$; the use of objects in the mouth and the chewing function $(\mathrm{p}=0.001)$; oral breathing, phonoarticulatory organs' appearance and posture $(\mathrm{p}<0.001)$ and swallowing function $(p=0.002)$. Besides, being breastfed for a longer time modified phonoarticulatory organs' appearance and posture $(\mathrm{p}=0.001)$ and breathing function $(p=0.005)$. Conclusion: Feeding habits,

\section{Introducción}

El hábito es un comportamiento adquirido que se instala por ser agradable o dar satisfacción y placer. Debido a la repetición continua, se automatiza, se perfecciona y se vuelve inconsciente. ${ }^{1}$

Los hábitos orales pueden tener como consecuencias alteraciones oclusales y afectar el equilibrio neuromuscular orofacial y el crecimiento craneofacial, dependiendo del tiempo, intensidad, frecuencia, duración y característica del objeto utilizado, como también la edad del sujeto en la época en que se instalan los hábitos. ${ }^{2,3}$ Estos incluyen: biberón, succión de chupete y digital, morder objetos, succión y mordida del labio, onicofagia; hábitos funcionales - deglución atípica, respiración oral, entre otros. ${ }^{4,5}$

Este tema es de interés para los profesionales de la salud y estudios que relacionan la forma de alimentación, el desarrollo de hábitos orales, la oclusión y condiciones dentales y la función orofacial, enfatizando los aspectos miofuncionales. ${ }^{6-8}$

La evaluación clínica se considera esencial para el diagnóstico de los disturbios miofuncionales. El protocolo Evaluación Miofuncional Orofacial con plying with solid external objects in the mouth, pacifier use and mouth breathing were associated with changes in articulatory organs' appearance and posture, as well as, stomatognathic functions.

Key words: Speech, Language and Hearing Sciences, Habits, Child, Preschool, Stomatognathic System

valores (AMIOFE) $)^{9}$ fue validado para niños y desarrollado para la evaluación de estructuras y funciones orofaciales, y se ha utilizado en la investigación de la influencia de los hábitos orales en el perfil miofuncional orofacial de niños. ${ }^{10}$ Este protocolo permite al examinador expresar numéricamente en una escala categórica su percepción de las características y comportamientos observados. ${ }^{9}$

Considerando el diagnóstico de los cambios en el sistema estomatognático, cuanto más tarde esto sucede, mayores son los efectos en el equilibrio de estructuras funcionales, de postura y morfológicas. ${ }^{11,12}$

La intervención precoz en la disfunción de la musculatura orofacial puede restablecer el potencial de crecimiento y de desarrollo adecuado y el equilibrio en el desarrollo craneofacial. $^{13}$ Así, conocer cuáles son estos cambios y poder intervenir en la eliminación de hábitos orales inadecuados para fomentar el restablecimiento $y$ orientación para adecuar el sistema estomatognático es de extremada importancia. Así, para eliminar estos hábitos siempre se necesitará un enfoque multidisciplinario, buscando obtener un control tanto para aspectos físicos como psicológicos del niño. ${ }^{14}$ 
Partiendo de lo expuesto, el objetivo de este estudio es verificar la influencia de hábitos orales en el perfil miofuncional orofacial de niños de tres a cinco años.

\section{Materiales y Métodos}

Este estudio se caracteriza por ser de carácter observacional y transversal. Se aplicó el protocolo para la evaluación del aspecto miofuncional orofacial y el cuestionario sobre los hábitos orales en 73 niños entre tres y cinco años de edad, que frecuentaron la Clínica Infantojuvenil de la Facultad de Odontología de la Universidad Federal de Rio Grande do Sul (UFRGS). Se excluyeron los niños que no completaron la evaluación fonoaudiológica y los que ya tuvieron o tienen una atención en el área fonoaudiológica u odontológica. Los padres o responsables firmaron el Consentimiento Informado, para ratificar el acuerdo con la participación. Los datos utilizados para este estudio forman parte de un proyecto de investigación previamente registrado y aprobado en el Comité de Ética en Investigación de la UFRGS con el nº 19236.

Para la evaluación fonoaudiológica se utilizó el protocolo AMIOFE, ${ }^{9}$ por tratarse de un instrumento nacional validado. Este protocolo es sensible para evaluar los desórdenes miofuncionales orofaciales, definidos por las autoras como alteraciones/disfunciones del aspecto, postura o movilidad, y de las funciones. Lo mismo consta en las siguientes evaluaciones:

- Aspecto y postura: labios, mandíbula, mejillas, lengua, paladar duro, rostro.
- Movilidad: se solicitó a los niños que hicieran por 5 segundos movimientos distintos de los labios, lengua, mandíbula y mejillas. Se atribuye un puntaje en la escala: $3=$ normal, cuando son movimientos precisos y sin temblores; $2=$ capacidad insuficiente, cuando falta precisión en los movimientos, hay temblores y movimientos asociados de otros componentes; $1=$ ausencia de capacidad o es incapaz de ejecutar la tarea.

- Funciones: respiración, considerada nasal cuando el sellamiento labial se realice sin esfuerzo durante el reposo; deglución, considerada normal cuando la lengua está contenida en la cavidad oral, hay contracción de los músculos elevadores y sellamiento labial anterior sin esfuerzo; masticación, se solicitó a los niños que masticaran de forma habitual y se consideró bilateral alternada o simultánea, unilateral crónica (95\% del mismo lado), anterior o no realiza la función. Además de lo que se describe en el protocolo, cada una de las funciones se consideró como normal (3), disfunción leve (2), y disfunción grave (1).

La evaluación miofuncional se aplicó con una fonoaudióloga mediante la observación indirecta, denominada antroposcopía. Se complementó por el análisis posterior de imagen registrada en cámara digital colocada a 1 metro de distancia delindividuo. Elniño permaneció sentado, con los pies apoyados en el suelo y con la cabeza en la posición habitual. La fonoaudióloga permaneció sentada frente al niño para la evaluación. Los pasos de la evaluación siguieron la orientación para aplicar el protocolo AMIOFE. ${ }^{9}$ Para 
evaluar la masticación y la deglución (sólido) se ofrecieron $25 \mathrm{~g}$ de pan francés y para la deglución de líquido $50 \mathrm{ml}$ de agua en un vaso de plástico transparente.

En el mismo momento que el niño estaba siendo evaluado, se aplicó el cuestionario sobre los hábitos orales, elaborado con base en la literatura, ${ }^{15}$ para obtener datos sobre la lactancia materna, hábitos orales (chupete, dedo, onicofagia, objetos en la boca, la respiración oronasal, biberón antes de dormir, biberón durante la noche), como características demográficas (edad y sexo) y socioeconómicas (ingresos familiares y escolaridad de los padres). La administración del cuestionario fue realizada por otra investigadora, que fue previamente entrenada para la lectura lenta y en entonación constante para aplicar cada cuestionario y opciones de respuestas. Las investigadoras hicieron evaluaciones ciegas.

Todos los pacientes que pasaron por la presente investigación y en los que se constataron alteraciones relacionadas con el aspecto fonoaudiológico fueron clasificados en el respectivo servicio, de acuerdo con la demanda.

Para verificar la influencia de los hábitos orales se consideraron: uso o no de biberón; uso o no de chupete; tener el hábito o no de succión digital; tener el hábito o no de colocar objetos en la boca (sistemático); tener el hábito o no de onicofagia; presentar o no respiración oronasal. Los hábitos orales fueron exclusivamente referidos por los padres, excepto la respiración oronasal que se verificó clínicamente a partir del propio protocolo de evaluación utilizado.
Para el análisis estadístico de los datos se utilizó el software (SPSS) v.18.0 Windows. Las variables cuantitativas se analizaron a través de la media (desviación estándar) o mediana (mínima y máxima) y las variables cualitativas a través de porcentaje relativo y absoluto. Para comparar promedios entre los grupos, se utilizó el test t-Student. El nivel de significación utilizado fue del $5 \%$.

\section{Resultados}

La muestra en estudio contaba inicialmente con 100 participantes, sin embargo $27(27 \%)$ se excluyeron, estos menores de 4 años de edad, no cumplieron los criterios de inclusión, por no completar todos los ítems del protocolo AMIOFE. El principal ítem de exclusión fue no realizar las tareas de movilidad.

En relación con la caracterización de la muestra, 39 (53,4\%) de los niños eran del sexo femenino, tenían en promedio edad de $4,7 \pm 0,8$ años. La lactancia sucedió en $67(91,8 \%)$ niños, con un promedio de 1 año (mínimo de 2 meses y máximo de 4 años y 1 mes). El tiempo promedio de amamantamiento materno exclusivo fue de 4,3 $\pm 1,9$ meses. Con respecto a la condición socioeconómica, los ingresos familiares de la mayoría, 50 (68,5\%), quedaron entre dos salarios mínimos.

La suma total del AMIOFE fue de 91,6 65,4 puntos (IC 95\%=90,3-92,9), con el mínimo de 75 y máximo de 100 puntos.

Los aspectos, las condiciones de postura y la movilidad de las estructuras orofaciales obtenidas por el AMIOFE están descritas en la Tabla 1. 
Tabla 1. Aspectos, condiciones de postura y movilidad de las estructuras orofaciales obtenidas por el AMIOFE en la muestra de niños entre tres a cinco años $(n=73)$

\begin{tabular}{|c|c|c|}
\hline Variables & $\begin{array}{c}\text { Adecuado } \\
\text { n (\%) }\end{array}$ & $\begin{array}{c}\text { Inadecuado } \\
\mathrm{n}(\%)\end{array}$ \\
\hline \multicolumn{3}{|l|}{ Aspectos y posturas } \\
\hline Postura del Labio & $42(57,5 \%)$ & $31(42,5 \%)$ \\
\hline Postura Vertical de la Mandíbula & $69(94,5 \%)$ & $4(5,5 \%)$ \\
\hline Aspecto de Mejillas & $68(93,2 \%)$ & $5(6,8 \%)$ \\
\hline Apariencia Facial & $59(80,8 \%)$ & $14(19,2 \%)$ \\
\hline Posición de la Lengua & $45(61,6 \%)$ & $28(38,4 \%)$ \\
\hline Aspecto del paladar duro & $66(90,4 \%)$ & $7(9,6 \%)$ \\
\hline \multicolumn{3}{|l|}{ Mobilidad } \\
\hline \multicolumn{3}{|l|}{ Labios } \\
\hline Protrusión & $71(97,3 \%)$ & $2(2,7 \%)$ \\
\hline Retrusión & $71(97,3 \%)$ & $2(2,7 \%)$ \\
\hline Lateralidad para Derecha & $26(35,6 \%)$ & $47(64,4 \%)$ \\
\hline Lateralidad para Izquierda & $22(30,1 \%)$ & $51(69,9 \%)$ \\
\hline \multicolumn{3}{|l|}{ Lengua } \\
\hline Protrusión & $67(91,8 \%)$ & $6(8,2 \%)$ \\
\hline Retrusión & $67(91,8 \%)$ & $6(8,2 \%)$ \\
\hline Lateralidad Derecha & $71(97,3)$ & $2(2,7 \%)$ \\
\hline Lateralidad Izquierda & $71(97,3)$ & $2(2,7 \%)$ \\
\hline Elevar & $60(82,2 \%)$ & $13(17,8 \%)$ \\
\hline Bajar & $72(98,6 \%)$ & $1(1,4 \%)$ \\
\hline \multicolumn{3}{|l|}{ Mandíbula } \\
\hline Apertura & $72(98,6 \%)$ & $1(1,4 \%)$ \\
\hline Cierre & $72(98,6 \%)$ & $1(1,4 \%)$ \\
\hline Lateralidad Derecha & $64(87,7 \%)$ & $9(12,3)$ \\
\hline Lateralidad Izquierda & $65(89,0 \%)$ & $8(11,0 \%)$ \\
\hline Protrusión & $67(91,8 \%)$ & $6(8,2 \%)$ \\
\hline \multicolumn{3}{|l|}{ Mejillas } \\
\hline Inflar & $71(97,3 \%)$ & $2(2,7 \%)$ \\
\hline Suflar & $68(93,2 \%)$ & $5(6,8 \%)$ \\
\hline Retraer & $62(84,9 \%)$ & $11(15,1 \%)$ \\
\hline Lateralizar & $57(78,1 \%)$ & $16(21,9 \%)$ \\
\hline
\end{tabular}


Tabla 2. Características de las funciones orofaciales obtenidas por el AMIOFE en la muestra de niños entre tres a cinco años $(n=73)$

\begin{tabular}{|c|c|}
\hline Variables & $\mathrm{n}(\%)$ \\
\hline \multicolumn{2}{|l|}{ Respiración } \\
\hline \multicolumn{2}{|l|}{ Modo de respiración } \\
\hline Nasal & $49(67,1 \%)$ \\
\hline Oral & $24(32,9 \%)$ \\
\hline \multicolumn{2}{|l|}{ Deglución } \\
\hline \multicolumn{2}{|l|}{ Comportamiento de los labios } \\
\hline Normal & $32(43,8 \%)$ \\
\hline Disfunción leve & $35(47,9 \%)$ \\
\hline Disfunción moderada & $5(6,8 \%)$ \\
\hline Disfunción severa & $1(1,4 \%)$ \\
\hline \multicolumn{2}{|l|}{ Comportamento del lengua } \\
\hline Normal & $41(56,2 \%)$ \\
\hline Adaptación a disfunción & $31(42,5 \%)$ \\
\hline Protrusión en exceso & $1(1,4 \%)$ \\
\hline \multicolumn{2}{|l|}{ Movimiento de la cabeza } \\
\hline Presente & $11(15,1 \%)$ \\
\hline Ausente & $62(84,9 \%)$ \\
\hline \multicolumn{2}{|l|}{ Tensión de los músculos faciales } \\
\hline Presente & $39(53,4 \%)$ \\
\hline Ausente & $34(46,6 \%)$ \\
\hline \multicolumn{2}{|l|}{ Eficiencia de deglución } \\
\hline \multicolumn{2}{|l|}{ Bolo sólido } \\
\hline No repite deglución del mismo bolo & $57(78,1 \%)$ \\
\hline Una repetición & $9(12,3 \%)$ \\
\hline Deglución múltiple & $7(9,6 \%)$ \\
\hline \multicolumn{2}{|l|}{ Bolo líquido } \\
\hline No repite deglución del mismo bolo & $72(98,6 \%)$ \\
\hline Una repetición & $1(1,4 \%)$ \\
\hline \multicolumn{2}{|l|}{ Masticación } \\
\hline \multicolumn{2}{|l|}{ Patrón de masticación } \\
\hline Bilateral alternada & $44(60,3 \%)$ \\
\hline Bilateral simultánea & $7(9,6 \%)$ \\
\hline Unilateral preferencial & $15(20,5 \%)$ \\
\hline Unilateral crónica & $6(8,2 \%)$ \\
\hline Anterior & $1(1,4 \%)$ \\
\hline \multicolumn{2}{|l|}{ Movimiento de la cabeza } \\
\hline Presente & $3(4,1 \%)$ \\
\hline Ausente & $70(95,9 \%)$ \\
\hline \multicolumn{2}{|l|}{ Postura alterada } \\
\hline Presente & $5(6,8 \%)$ \\
\hline Ausente & $68(93,2 \%)$ \\
\hline \multicolumn{2}{|l|}{ Escape de alimento } \\
\hline Presente & $4(5,5 \%)$ \\
\hline Ausente & $69(94,5 \%)$ \\
\hline
\end{tabular}

Las características de las funciones orofaciales obtenidas por el AMIOFE en la muestra de niños están descriptas en la Tabla 2.

Considerando los hábitos orales analizados, $64 \quad(87,7 \%)$ niños usaron biberón, entre los que 47 usaron tetina común $(64,4 \%)$. El promedio de edad de inicio del uso del biberón fue de 4 meses (mínimo de 0 meses y máximo de 3 años) y el promedio del tiempo de uso fue de 3 años y 3 meses. En el momento de la evaluación, el 53,3\% de los niños mantenían tal práctica.

Con respecto al chupete, 39 (53,4\%) niños lo usaron, 27 (37\%) utilizaron la tetina de tipo común. El uso de este artefacto inició antes del primer mes de vida y el promedio de término fue de 3 años y 8 meses. Menos evidente aparece el hábito de succión digital, en solo 7 (9,6\%). El promedio de edad de inicio de este hábito fue de 5 meses y el promedio de la edad de término 3 años y 9 meses.

Relataron los padres que $28(38,4 \%)$ niños permanecían con la boca abierta durante el día, sin embargo 48 (65,8\%) presentan este hábito durante la noche. Se destaca que la presencia de respiración oronasal se indicó con el protocolo del AMIOFE, analizada durante el reposo y en la propia evaluación miofuncional $24 \quad(32,9 \%)$ presentaron respiración oronasal.

La Tabla 3 presenta la relación entre los resultados del protocolo AMIOFE con los hábitos orales. Considerando los datos de caracterización de la muestra, haber amamantado en el seno materno por mayor tiempo influyó en la postura y aspecto de los órganos fonoarticulatorios, como también en la función de la respiración. 
Tabla 3. Relación entre los resultados del protocolo AMIOFE con los hábitos orales en 73 niños de tres a cinco años

\begin{tabular}{|c|c|c|c|c|c|c|c|}
\hline Variables & $\begin{array}{l}\text { Aspectos y } \\
\text { Posturas }\end{array}$ & Mobilidad & Respiración & $\begin{array}{l}\text { Funciones } \\
\text { Masticación }\end{array}$ & Deglución & $\begin{array}{l}\text { Total de las } \\
\text { Funciones }\end{array}$ & $\begin{array}{l}\text { Total del } \\
\text { AMIOFE }\end{array}$ \\
\hline \multicolumn{8}{|l|}{ Biberón } \\
\hline $\mathrm{Si}$ & $16,7 \pm 1,3$ & $53,4 \pm 4$ & $2,6 \pm 0,5$ & $6,1 \pm 1,3$ & $12,5 \pm 1,9$ & $21,2 \pm 2,9$ & $91,4 \pm 5,8$ \\
\hline No & $17,6 \pm 0,7$ & $53,7 \pm 2,2$ & $2,9 \pm 0,3$ & $5,6 \pm 1,9$ & $13,6 \pm 1,7$ & $22,1 \pm 2,4$ & $93,5 \pm 3,3$ \\
\hline $\mathrm{p}$ & $0,014^{*}$ & 0,81 & 0,14 & 0,34 & 0,12 & 0,41 & 0,31 \\
\hline \multicolumn{8}{|l|}{ Chupete } \\
\hline $\mathrm{Si}$ & $16,3 \pm 1,3$ & $53,2 \pm 4,3$ & $2,5 \pm 0,5$ & $5,9 \pm 1,4$ & $12,1 \pm 2$ & $20,6 \pm 3,1$ & $90,1 \pm 5,8$ \\
\hline No & $17,4 \pm 0,9$ & $53,7 \pm 3,2$ & $2,8 \pm 0,4$ & $6,2 \pm 1$ & $13,3 \pm 1,6$ & $22,3 \pm 2$ & $93,5 \pm 4,5$ \\
\hline $\mathrm{p}$ & $<0,001^{*}$ & 0,55 & $0,04^{*}$ & 0,44 & $0,006^{*}$ & $0,008^{*}$ & $0,007^{*}$ \\
\hline \multicolumn{8}{|c|}{ Succión digital } \\
\hline $\mathrm{Si}$ & $16,7 \pm 1,2$ & $52,7 \pm 4,6$ & $2,8 \pm 0,4$ & $5,8 \pm 1,2$ & $11,4 \pm 2,5$ & $20,1 \pm 3,2$ & $89,6 \pm 8,1$ \\
\hline No & $16,8 \pm 1,3$ & $53,5 \pm 3,8$ & $2,6 \pm 0,5$ & $6,1 \pm 1,3$ & $12,7 \pm 1,8$ & $21,5 \pm 2,7$ & $91,8 \pm 5,1$ \\
\hline $\mathrm{p}$ & 0,79 & 0,59 & 0,21 & 0,08 & 0,68 & 0,23 & 0,49 \\
\hline \multicolumn{8}{|l|}{ Objetos } \\
\hline $\mathrm{Si}$ & $17,1 \pm 1$ & $53,8 \pm 3$ & $2,7 \pm 0,5$ & $6,7 \pm 0,6$ & $13,5 \pm 1,9$ & $22,9 \pm 2$ & $93,6 \pm 4,5$ \\
\hline No & $16,8 \pm 1,3$ & $53,4 \pm 4$ & $2,7 \pm 0,5$ & $5,8 \pm 1,3$ & $12,4 \pm 1,9$ & $21 \pm 2,8$ & $91,1 \pm 5,6$ \\
\hline $\mathrm{p}$ & 0,32 & 0,89 & 0,7 & $0,001^{*}$ & 0,06 & $0,018^{*}$ & 0,12 \\
\hline \multicolumn{8}{|c|}{ Onicofagia } \\
\hline $\mathrm{Si}$ & $17,3 \pm 1,1$ & $51,7 \pm 5,6$ & $2,7 \pm 0,5$ & $6,3 \pm 1,1$ & $13 \pm 1,8$ & $22 \pm 2,4$ & $91,2 \pm 5,8$ \\
\hline No & $16,7 \pm 1,3$ & $53,7 \pm 3,7$ & $2,6 \pm 0,5$ & $5,9 \pm 1,3$ & $12,5 \pm 1,9$ & $21,2 \pm 2,9$ & $91,7 \pm 5,4$ \\
\hline $\mathrm{p}$ & 0,09 & 0,07 & 0,57 & 0,41 & 0,44 & 0,31 & 0,73 \\
\hline \multicolumn{8}{|c|}{$\begin{array}{l}\text { Respiración } \\
\text { oronasal }\end{array}$} \\
\hline $\mathrm{Si}$ & $16,5 \pm 1,3$ & $53 \pm 4,1$ & $2,5 \pm 0,5$ & $5,9 \pm 1,3$ & $12,2 \pm 1,9$ & $20,7 \pm 2,8$ & $90,2 \pm 5,3$ \\
\hline No & $17,6 \pm 0,7$ & $54,6 \pm 2,8$ & $3 \pm 0$ & $6,3 \pm 1$ & $13,7 \pm 1,6$ & $23 \pm 2$ & $95,2 \pm 4$ \\
\hline $\mathrm{p}$ & $<0,001^{*}$ & 0,09 & $<0,001^{*}$ & 0,29 & $0,002^{*}$ & $0,001^{*}$ & $<0,001^{*}$ \\
\hline
\end{tabular}

* $\mathrm{p}<0,05$ - Teste $t$ de Student

\section{Discusión}

En la muestra estudiada, se observó que los hábitos orales están presentes con alta frecuencia, ${ }^{16,17}$ y los de mayor prevalencia son los hábitos de chupete y biberón. 8, 16-20

Los hábitos orales merecen más atención siempre que perduren o se manifiesten en niños con edad superior a tres años. Se sabe que puede suceder una autocorrección de alguna desarmonía oclusal y muscular causada por el hábito, si se mantiene por un periodo menor o igual a tres años; $y$ que a partir de esa edad, podrá causar alteraciones orofaciales comprometedoras del crecimiento facial armónico. ${ }^{17,21}$

Algunos trabajos observaron las consecuencias del biberón y el chupete en el desarrollo orofacial. 17,22,23 Estos verificaron una alteración en la oclusión labial debido a la acción ineficiente de la musculatura involucrada, como también 
de postura habitual atípica de la lengua con señales de flacidez y protrusión. Además, indican alteraciones de arcos dentales con consecuencias en la oclusión y en la articulación de los fonemas. En ese estudio estos hábitos demostraron una relación con el aspecto y postura de los órganos fonoarticulatorios.

El biberón es un hábito oral frecuente en niños, ${ }^{18,24}$ con gran prevalencia de su uso en los primeros años de vida, ${ }^{19,23}$ lo que también se observó en este estudio. Este hábito surgiría como consecuencia del destete precoz, reemplazando la lactancia natural. ${ }^{8}$ Una investigación que evaluó a niños con cuatro años de edad que usaban biberón, observó un sellamiento labial en un $65 \%$ de los niños, sin embargo el 53\% presentaban una postura inadecuada de la lengua. ${ }^{22}$ Otros estudios observaron que el uso de ese artefacto causó la pérdida del sellamiento labial; además, favoreció una posición inadecuada de la lengua y alteró la forma de la mandíbula. ${ }^{6,25}$

Con respecto al uso de chupete, los resultados fueron similares a los de otros autores, estaba presente en más de la mitad de los evaluados y se daba el uso de la tetina de tipo común en su mayoría. ${ }^{17,18,24}$ En otra investigación, sucedió la asociación significativa entre la postura habitual de lengua y el uso del chupete, y correspondía la postura más posterior a la población infantil que no hacía uso del chupete. ${ }^{23}$

Estudios observaron que el hábito oral de succión no nutritiva (chupete o dedo) presentó una asociación perjudicial directa e indirecta sobre las estructuras y funciones estomatognáticas. ${ }^{19,26}$ Cuando un niño hace un uso frecuente de chupete, probablemente presentará una respiración oral debido a una postura muscular facial y lingual compensatoria hipotónica, lo que interfiere aún más en los mecanismos de lactancia materna. ${ }^{27}$ En ese estudio, el hábito de succión del chupete estuvo relacionado con el patrón de respiración y deglución de los niños. En lo que se refiere a la succión digital el resultado de este estudio se asemeja a otras investigaciones en la población infantil. ${ }^{8,18,24}$

Niños con respiración nasal presentan un patrón de respiración normal y son amamantados por un periodo más largo que los niños con respiración oral, ${ }^{18,25,28}$ corroborando los descubrimientos de este estudio que verificó la relación de la función respiración con el tiempo de lactancia materna. Por otro lado, un estudio no observó diferencia entre respiradores oronasales y respiradores nasales y el tiempo de lactancia materna. Observaron que en el primer grupo, el $62,3 \%$ amamantados en el seno por un periodo menor de 4 meses y el $37 \%$ por un tiempo mayor o igual a 4 meses y en el segundo grupo, el 60,6\% amamantaron por menos de 4 meses y el $39,4 \%$ por tiempo mayor o igual a 4 meses. ${ }^{29}$

La postura y aspectos de los órganos fonoarticulatorios y el tiempo de amamantamiento materno indicaron una relación significativa, corroborando los descubrimientos de otras investigaciones. ${ }^{10,19,23}$ Estudios observaron que la ausencia de no sellamiento labial ocurrió en niños que amamantaron exclusivamente en los primeros seis meses de vida y que la probabilidad de desarrollar un patrón de respiración nasal aumentó de acuerdo con el aumento de la duración de la lactancia materna. 19,28 $^{2}$

La literatura describe significativa 
asociación entre la presencia de hábitos orales, principalmente hábitos de succión y hábitos de mordida, en respiradores oronasales. ${ }^{22}$ De acuerdo con lo encontrado en este trabajo se observó una similar frecuencia de niños con el hábito de permanecer con la boca abierta durante la noche y la relación de este hábito con el uso de la mamadera, del chupete y de succión digital; sin embargo, tal relación no fue significativa. ${ }^{18}$

Una investigación con niños respiradores oronasales verificó una postura caracterizada por ausencia de oclusión labial en el 77,5\% de la muestra, tonicidad de lengua reducida en un $80 \%$ y paladar duro estrecho en un $42,5 \%$ de los niños. ${ }^{30}$

Alteraciones en los órganos fonoarticulatorios, como postura y tonicidad, pueden estar relacionadas con disturbios en la función de respiración ${ }^{29,30}$ y en la función de deglución, lo que podría explicar la relación entre esos factores. Al abrir la boca para respirar, suceden adaptaciones y desequilibrio de las estructuras y funciones orofaciales que comprometen esta función, generando dificultad en la alimentación y perjudicando directamente el estado de nutrición del individuo. ${ }^{29}$

Los hábitos orales, principalmente el biberón, chupete y la respiración oronasal se relacionaron con alteraciones de la motricidad orofacial incluso el sistema estomatognático y sus funciones. Aunque no haya sido el objetivo de este estudio, se observó que el mayor tiempo de lactancia benefició aspectos y posturas de los órganos fonoarticulatorios y la función respiración.

Teniendo en cuenta la significativa prevalencia de los hábitos orales en esa franja de edad, ${ }^{8,16-20}$ se refuerza la preocupación con respecto a la influencia que estos pueden traerle al sistema estomatognático. Se observa la necesidad de continuar la producción de conocimientos sobre formas eficaces de intervención en la familia. La importancia de la participación de diferentes áreas del conocimiento, como la odontopediatría y la fonoaudiología, principalmente en el sentido de proponerse formas de intervenciones precoces, es de extrema importancia para el desarrollo adecuado de los niños.

\section{Conclusión}

En el presente estudio, los hábitos de biberón, objetos en la boca, chupete y respiración oral demostraron una relación con las alteraciones en el sistema estomatognático y en sus funciones. Estos descubrimientos justifican la importancia de un enfoque multidisciplinario, a fin de minimizar el impacto de las alteraciones miofuncionales orofaciales y maximizar la calidad de vida de esos niños.

\section{Referencias bibliográficas}

1. Vasconcelos FMN, Massoni, ACLT, Ferreira AMB, Katz RT, Rosenblat A. Ocorrência de Hábitos Bucais Deletérios em Crianças da Região Metropolitana do Recife, Pernambuco, Brasil. Pesq Bras Odontoped Clin Integr. 2009; 9: 327-32.

2. Fofano CSN, Mialhe FL, Silva RP, Brum SC. Conhecimentos, atitudes e práticas maternas em relação ao uso da chupeta. Pesq Bras Odontoped Clin Integr. 2009; 9:119-23. 
3. Gonçalves LPV, Toledo OA, Otero SAM. Relação entre bruxismo, fatores oclusais e hábitos bucais. Dental Press J. Orthod. 2010; 15: 97-104.

4. Vasconcelos FMN, Massoni ACL, Heimer MV, Ferreira AMB, Katz CRT, Rosemblatt A. Non-Nutritive sucking habits, anterior open bite and associated factors in Brazilian children aged 30-59 months. Braz Dent J. 2011; 22:140-5.

5. Silvério KCA, Ferreira APS, Johanns CM, Wolf A, Furkim AM, Marques JM. Relação escolaridade, faixa etária e profissão da mães com a oferta de chupeta e mamadeira a seus filhos. Rev CEFAC. 2012; 14:610-5.

6. Santos-Neto ET, Barbosa RW, Oliveira AE, Zandonade E. Fatores associados ao surgimento da respiração bucal nos primeiros meses do desenvolvimento infantil. Rev Bras Crescimento Desenvolv Hum. 2009; 19:237-248.

7. Leite-Cavalcanti A, Medeiros-Bezerra PK, Moura C. Aleitamento natural, aleitamento artificial, hábitos de sucção e maloclusões em pré-escolares brasileiros. Rev Salud Pública. 2007; 9:194-204.

8. Almeida MEC de, Melo NS, Maia SA, Costa AMM da, Souza KR. A influência do desmame precoce no desenvolvimento de hábitos bucais deletérios. ConScientiae Saúde. 2007; 6:227-34.

9. Felício CM, Ferreira CLP. Protocol of orofacial myofunctional evaluation with scores. Inter J Ped Otorhinolaryngol. 2008; 72:367-75.

10. Medeiros APM, Ferreira JTL, Felício CM de. Correlação entre métodos de aleitamento, hábitos de sucção e comportamentos orofaciais. Pró-Fono. 2009; 21:315-9.

11. Cattoni DM, Fernandes FD, Francesco RC Di, Latorre MRDO. Características do sistema estomatognático de crianças respiradoras orais: enfoque antroposcópico. Pró-Fono. 2007; 19:347-51.

12. Whitaker ME, Junior AST, Genaro KF. Protocolo de Avaliação Clínica da Função Mastigatória. Rev CEFAC. 2009; 11:311-23.

13. Degan VV, Puppin-Rontani RM. Remoção de hábitos e terapia miofuncional: restabelecimento da deglutição e repouso lingual. Pró-Fono. 2005; 17:375-82.

14. Pizzol KEDC, Montanh SS, Fazan ET, Boeck EM, Rastelli ANS: Prevalence of nonnutritive sucking habits and their relationshipto age, gender and type of feeding in preschool children from Araraquara-Brazil. Rev CEFAC. 2012; 3:506-515.

15. Hallet KB, O'Rourke PK. Pattern and severity of early childhood caries. Community Dent Oral Epidemiol. 2006; 34:25-35.

16. Zapata M, Bachiega JC, Marangoni AF, Jeremias JEM, Ferrari RAM, Bussadori SK et al. Mordida aberta e hábitos orais deletérios. Rev CEFAC. 2010; 12:267-71.

17. Pereira TS, Oliveira F, Cardoso MCAF. Associação entre hábitos orais deletérios e as estruturas e funções do sistema estomatognático: percepção dos responsáveis. CoDAS. 2017;29(3):e20150301.

18. Lima GN, Cordeiro CM, Justo JS, Rodrigues LCB. Mordida aberta anterior e hábitos orais em crianças. Rev Soc Bras Fonoaudiol. 2010; 15:369-75.

19. Lopes TSP, Moura LFAD, Lima MCMP. Association between breastfeeding and breathing pattern in children: a sectional study. Jornal de Pediatria. 2014; 90:396-402.

20. Garbin CAS, Garbin AJÍ, Martins RJ, de Souza NP, Moimaz SAS. Prevalence of non-nutritive sucking habits in preschoolers and parents' perception of its relationship with malocclusions. Cien Saude Colet. 2014;19(2):553-8.

21. Verrastro AP, Tashima AY, Ideriha PN, Stefani FM, Rodrigues CRMD, Wanderley MT. Características oclusais e miofuncionais orais das crianças atendidas na Clinica de Odontopediatria da Faculdade de Odontologia da USP. Revista Inst Ciênc Saúde. 2009; 27:394-9.

22. Carrascoza KC, Possobon RF, Tomita LM, Moraes ABA de. Bottle-feeding and oral facial development. J Pediatr. 2006; 82:395-8.

23. Araújo CMT, Silva GAP, Coutinho SB. A Utilização da Chupeta e o Desenvolvimento Sensório Motor Oral. Rev CEFAC. 2009; 11:261-7.

24. Pires SC, Giugliani ERJ, Silva FC. Influence of the duration of breastfeeding on quality of muscle function during mastication in preschoolers: a cohort study. BMC Public Health. 2012; 12:934.

25. Da Costa SP, van der Schans CP, Boelema SR, van der Meij E, Boerman MA, Bos AF. Sucking patterns in fullterm infants between birth and 10 weeks of age. Infant Behav Dev. 2010; 33:61-7.

26. Nieuwenhuis T, Da Costa SP, Bilderbeek E, Geven WB, Van der Schans CP, Bos AF. Uncoordinated 
sucking patterns in preterm infants are associated with abnormal general movements. J Pediatr. 2012; 161:792-8.

27. Moimaz SA, Rocha NB, Garbin AJ, Saliba O. A influência da prática do aleitamento materno na aquisição de hábitos de sucção não nutritivos e prevenção de oclusopatias. Rev Odontol UNESP. 2013; 42:31-6.

28. Limeira AB, Aguiar CM, Bezarra NS, Câmara AC. Association between breastfeeding and the development of breathing patterns in children. Eur J Pediatr. 2013; 172:519-524.

29. Cunha DA, Silva GAP, Motta MEFA, Lima CR, Silva HJ. Estado nutricional em respiradores orais. Rev CEFAC. 2007;9:47-54.

30. Andrade FV. Andrade DV, Araújo AS, Ribeiro ACC, Deccax LDG, Nemr, K. Alterações estruturais de órgãos fonoarticulatórios e más oclusões dentárias em respiradores orais de 6 a 10 anos. Rev CEFAC. 2005; 7:318-25.

Recibido: 09/03/17

Aceptado: 10/09/19

Correspondencia: Mônica Carminatti, e-mail:moni.carminatti@hotmail.com 